\title{
Regular character tables of symmetric groups
}

\author{
Jørn B. Olsson \\ Matematisk Afdeling \\ Universitetsparken 5, 2100 Copenhagen, Denmark \\ olsson@math.ku.dk
}

Submitted: Apr 17, 2002; Accepted: Apr 15, 2003; Published: Apr 23, 2003

MR Subject Classification: 20C30

\begin{abstract}
We generalize a well-known result on the determinant of the character tables of finite symmetric groups.
\end{abstract}

It is a well-known fact that if $X_{n}$ is the character table of the symmetric group $S_{n}$, then the absolute value of the determinant of $X_{n}$ equals $a_{n}$, which is defined as the product of all parts of all partitions of $n$. It also equals $b_{n}$, which is defined as the product of all factorials of all multiplicities of parts in partitions of $n$. Proofs of this may be found in [6], [5]. We sketch a proof below.

In this brief note we present generalizations of this to certain submatrices of $X_{n}$ (called regular/singular character tables). We get such character tables for each choice of an integer $\ell \geq 2$. This is a perhaps slightly surprising consequence of results in [4]. The above result is obtained when we choose $\ell \geq n$.

If $\mu$ is a partition of $n$ we write $\mu \vdash n$ and then $z_{\mu}$ denotes the order of the centralizer of an element of (conjugacy) type $\mu$ in $S_{n}$. Suppose $\mu=\left(1^{m_{1}}, 2^{m_{2}}, \ldots\right)$, is written in exponential notation. Then we may factor $z_{\mu}=a_{\mu} b_{\mu}$, where

$$
a_{\mu}=\prod_{i \geq 1} i^{m_{i}}, \quad b_{\mu}=\prod_{i \geq 1} m_{i} !
$$

We define

$$
a_{n}=\prod_{\mu \vdash n} a_{\mu}, \quad b_{n}=\prod_{\mu \vdash n} b_{\mu} .
$$

Proposition 1: We have that $\left|\operatorname{det}\left(X_{n}\right)\right|=a_{n}=b_{n}$.

Proof: (See also [6].) By column orthogonality for the irreducible characters of $S_{n}, X_{n}^{t} X_{n}$ is a diagonal matrix with the integers $z_{\mu}, \mu \vdash n$ on the diagonal. It follows that in the 
above notation $\operatorname{det}\left(X_{n}\right)^{2}=\prod_{\mu \vdash n} z_{\mu}=a_{n} b_{n}$. By [2], Corollary 6.5 we have $\left|\operatorname{det}\left(X_{n}\right)\right|=a_{n}$. The result follows.

Another proof of the fact that $a_{n}=b_{n}$ for all $n$ may be found in [3].

We choose an integer $\ell \geq 2$, which is fixed from now on. Several concepts below, like regular, singular, defect etc. refer to the integer $\ell$.

A partition is called regular if no part is repeated $\ell$ or more times and is called class regular, if no part is divisible by $\ell$. A partition which is not regular (class regular) is called singular (class singular). We let $p(n)$ be the number of partitions of $n$. The number $p^{*}(n)$ of regular partitions of $n$ equals the number of class regular partitions of $n$ and then $p^{\prime}(n)=p(n)-p^{*}(n)$ is the number of (class)singular partitions of $n$. The irreducible characters and the conjugacy classes of $X_{n}$ are labelled canonically by the partitions of $n$. An irreducible character is called regular (singular), if the partition labelling it is regular (singular). A conjugacy class is called regular (singular), if the partition labelling it is class regular (class singular). The regular character table $X_{n}^{\text {reg }}$ contains the values of regular characters on regular classes and the singular character table $X_{n}^{\text {sing }}$ is defined analogously.

Let

$$
a_{n}^{\text {creg }}=\prod_{\mu \text { class regular }} a_{\mu}, \quad b_{n}^{\text {creg }}=\prod_{\mu \text { class regular }} b_{\mu}
$$

and define $a_{n}^{\text {csing }}$ and $b_{n}^{\text {csing }}$ correspondingly such that $a_{n}$ and $b_{n}$ are factored into a "regular" and a "singular" component, $a_{n}=a_{n}^{\text {creg }} a_{n}^{\text {csing }}, b_{n}=b_{n}^{\text {creg }} b_{n}^{\text {csing }}$.

Our main results are:

Theorem 2: The regular character table satisfies: $\left|\operatorname{det}\left(X_{n}^{\mathrm{reg}}\right)\right|=a_{n}^{\mathrm{creg}}$.

Theorem 3: The singular character table satisfies: $\left|\operatorname{det}\left(X_{n}^{\text {sing }}\right)\right|=b_{n}^{\text {csing }}$.

Remark: In the case where $\ell=p$ is a prime number, we have that the absolute value of the determinant of the Brauer character table of $S_{n}$ in characteristic $p$ is also $a_{n}^{\text {creg }}$.

When $\mu \vdash n$, say $\mu=\left(i^{m_{i}(\mu)}\right)$ we define the defect of $\mu$ by

$$
d_{\mu}=\sum_{i, j \geq 1}\left\lfloor\frac{m_{i}(\mu)}{\ell^{j}}\right\rfloor,
$$

where $\lfloor\cdot\rfloor$ means "integral part of."

We start the proof of Theorems 2 and 3 with a key result which may be of independent interest. It generalizes the identity $a_{n}=b_{n}$ above and is obtained by modifying an idea implicit in [6], see also [7], Exercise 26, p.48 and p.59. An unpublished note of John Graham communicated to the author by Gordon James has been useful. The case where $\ell$ is a prime is implicit in [5], where proofs are based on modular representation theory.

Theorem 4: We have that $b_{n}^{\text {creg }} / a_{n}^{\text {creg }}=\ell^{c_{n}}$, where

$$
c_{n}=\sum_{\mu \text { class regular }} d_{\mu}
$$


Proof: Consider the set $\mathcal{T}$ of triples

$$
\mathcal{T}=\left\{(\mu, i, j) \mid \mu \text { class regular, } i, j \geq 1, m_{i}(\mu) \geq j\right\}
$$

We claim that

$$
a_{n}^{\text {creg }}=\prod_{(\mu, i, j) \in \mathcal{T}} i, \quad b_{n}^{\text {creg }}=\prod_{(\mu, i, j) \in \mathcal{T}} j .
$$

Indeed, for a fixed class regular $\mu$ and a fixed non-zero block $i^{m_{i}(\mu)}$ in $\mu$, the elements $(\mu, i, 1),(\mu, i, 2), \cdots,\left(\mu, i, m_{i}(\mu)\right)$ are precisely the ones in $\mathcal{T}$ starting with $\mu$ and $i$. These elements give a contribution $i^{m_{i}(\mu)}$ to $a_{n}^{\text {creg }}$ and a contribution $m_{i}(\mu)$ ! to $b_{n}^{\text {creg }}$.

We define an involution $\iota$ on $\mathcal{T}$ as follows. If $(\mu, i, j) \in \mathcal{T}$ then $\ell$ does not divide $i$, since $\mu$ is class regular. Also note that $\mu$ contains at least $j$ parts equal to $i$. Write $j=\ell^{v} j^{\prime}$, where $v$ is a non-negative integer and $\ell \nmid j^{\prime}$. We refer then to $j^{\prime}$ as the $\ell^{\prime}$-part of $j$. Let $\mu_{(i, j)}$ be obtained from $\mu$ by replacing $j$ parts equal to $i$ in $\mu$ by $\ell^{v} i$ parts equal to $j^{\prime}$. Then $\iota(\mu, i, j)$ is defined as $\left(\mu_{(i, j)}, j^{\prime}, \ell^{v} i\right)$, an element of $\mathcal{T}$. It is easily checked that $\iota^{2}$ is the identity.

This shows that

$$
a_{n}^{\text {creg }}=\prod_{(\mu, i, j) \in \mathcal{T}} i=\prod_{(\mu, i, j) \in \mathcal{T}} j^{\prime},
$$

where as above $j^{\prime}$ is the $\ell^{\prime}$-part of $j$. Thus $b_{n}^{\text {creg }} / a_{n}^{\text {creg }}=\ell^{c}$, where $c$ is the sum of the exponents of the powers $\ell^{v}$ of $\ell$, occuring as factors in the integers of the product $\prod_{\mu \text { class }, i \geq 1} m_{i}(\mu)$ !. If $m$ is a positive integer, then there are $\lfloor m / \ell\rfloor$ numbers among $1, \cdots, m$ which are divisible by $\ell,\left\lfloor m / \ell^{2}\right\rfloor$ numbers divisible by $\ell^{2}$, etc., giving a total exponent $\sum_{j \geq 1}\left\lfloor m / \ell^{j}\right\rfloor$ of $\ell$ in $m$ !. Applying this fact to each $m_{i}(\mu)$, we get our result.

Let $\chi_{\lambda}$ denote the irreducible character of $S_{n}$, labelled by the partition $\lambda \vdash n$, and $\chi_{\lambda}^{0}$ the restriction of $\chi_{\lambda}$ to the regular classes of $S_{n}$. In [4], Section 4, it was shown that there exist integers $d_{\lambda \rho}$ such that for each irreducible character $\chi_{\lambda}$ we have

$$
\chi_{\lambda}^{0}=\sum_{\rho \text { regular }} d_{\lambda \rho} \chi_{\rho}^{0}
$$

It follows from (1) that for any $\lambda$ the character

$$
\psi_{\lambda}=\chi_{\lambda}-\sum_{\rho \text { regular }} d_{\lambda \rho} \chi_{\rho}
$$

vanishes on all regular classes.

Proof of Theorem 2: The matrix form of (1) above may be stated as

$$
Y_{n}=D_{n} X_{n}^{\mathrm{reg}}
$$

where $Y_{n}$ is the $p(n) \times p^{*}(n)$-submatrix of $X_{n}$ containing the values of all irreducible characters on regular classes, and $D_{n}=\left(d_{\lambda \rho}\right)$ is the "decomposition matrix". Consider 
the corresponding "Cartan matrix" $C_{n}=\left(D_{n}\right)^{t} D_{n}$. (For an explanation of the terms decomposition matrix and Cartan matrix we refer to [4].)

Column orthogonality shows that

$$
\left(Y_{n}\right)^{t} Y_{n}=\left(X_{n}^{\mathrm{reg}}\right)^{t} C_{n} X_{n}^{\mathrm{reg}}=\Delta\left(z_{\mu}\right) .
$$

Here $\Delta$ is a diagonal matrix. Taking determinants we see that

$$
\operatorname{det}\left(X_{n}^{\mathrm{reg}}\right)^{2} \operatorname{det}\left(C_{n}\right)=\prod_{\mu \text { class regular }} z_{\mu}=a_{n}^{\text {creg }} b_{n}^{\text {creg }}
$$

By Proposition 6.11 in [4] (see also [1], Theorem 3.3) we have that $\operatorname{det}\left(C_{n}\right)=\ell^{c_{n}}$. It follows then from Theorem 4 that

$$
\operatorname{det}\left(C_{n}\right)=b_{n}^{\text {creg }} / a_{n}^{\text {creg }} .
$$

From (3) and (4) we conclude $\left|\operatorname{det}\left(X_{n}^{\text {reg }}\right)\right|=a_{n}^{\text {creg }}$, which proves the theorem.

Proof of Theorem 3: We assume that the rows and columns of $X_{n}$ are ordered such that the regular characters and classes are the first. Then the submatrix consisting of the intersection of the first $p^{*}(n)$ rows and the first $p^{*}(n)$ columns in $X_{n}$ is exactly $X_{n}^{\text {reg }}$. In fact $X_{n}$ has a block form

$$
X_{n}=\left[\begin{array}{cc}
X_{n}^{\mathrm{reg}} & A_{n} \\
B_{n} & X_{n}^{\text {sing }}
\end{array}\right] .
$$

We do some row operations on $X_{n}$ to get a new matrix $\bar{X}_{n}$ as follows: For each singular partition $\lambda^{\prime}$ and each regular partition $\rho$, subtract $d_{\lambda^{\prime} \rho}$ times the row labelled by $\rho$ from the row labelled by $\lambda^{\prime}$. Thus in $\bar{X}_{n}$ the row labelled by the singular partition $\lambda^{\prime}$ contains the values of the character $\psi_{\lambda^{\prime}}$ on all conjugacy classes. Since $\psi_{\lambda^{\prime}}$ vanishes on regular classes $\bar{X}_{n}$ looks like this:

$$
\bar{X}_{n}=\left[\begin{array}{cc}
X_{n}^{\mathrm{reg}} & A_{n} \\
0 & Q_{n}
\end{array}\right]
$$

for a suitable square $p^{\prime}(n)$-matrix $Q_{n}$. We have then $\operatorname{det}\left(X_{n}\right)=\operatorname{det}\left(\bar{X}_{n}\right)=\operatorname{det}\left(X_{n}^{\text {reg }}\right) \operatorname{det}\left(Q_{n}\right)$, whence by Theorem 2

$$
\operatorname{det}\left(Q_{n}\right)=a_{n}^{\mathrm{csing}}
$$

We now have that if $\lambda^{\prime}, \lambda^{\prime \prime}$ are singular partitions, then since $\psi_{\lambda^{\prime}}$ vanishes on regular classes

$$
<\psi_{\lambda^{\prime}}, \chi_{\lambda^{\prime \prime}}>=\sum_{\mu} \frac{1}{z_{\mu}} \psi_{\lambda^{\prime}}\left(x_{\mu}\right) \chi_{\lambda^{\prime \prime}}\left(x_{\mu}\right)=\sum_{\mu^{\prime} \text { class singular }} \frac{1}{z_{\mu^{\prime}}} \psi_{\lambda^{\prime}}\left(x_{\mu^{\prime}}\right) \chi_{\lambda^{\prime \prime}}\left(x_{\mu^{\prime}}\right) .
$$

Here $x_{\mu}$ is an element in the conjugacy class labelled by $\mu$. On the other hand by (2) $<\psi_{\lambda^{\prime}}, \chi_{\lambda^{\prime \prime}}>=\delta_{\lambda^{\prime} \lambda^{\prime \prime}}$. Translating these equations in terms of matrices

$$
Q_{n} \Delta\left(\frac{1}{z_{\mu^{\prime}}}\right)\left(X_{n}^{\text {sing }}\right)^{t}=E .
$$


Here again $\Delta$ is a diagonal matrix and $E$ is a $p^{\prime}(n)$-square identity matrix. Taking determinants

$$
\operatorname{det}\left(X_{n}^{\text {sing }}\right) \operatorname{det}\left(Q_{n}\right)=\prod_{\mu^{\prime}} \prod_{\text {class singular }} z_{\mu^{\prime}}=a_{n}^{\text {csing }} b_{n}^{\text {csing }}
$$

Now Theorem 3 follows from (5) and (6).

It should be remarked that Theorems 2 and 3 also hold, if we replace the irreducible characters $\chi_{\lambda}$ by the Young characters $\eta_{\lambda}$.

Acknowledgements: The author thanks C. Bessenrodt and M. Schocker for discussions. This research was supported by the Danish Natural Science Foundation.

\section{References}

[1] C. Bessenrodt, J.B.Olsson, A note on Cartan matrices for symmetric groups, preprint 2001. To appear in Arch. Math.

[2] G. James, The representation theory of the symmetric groups, Lecture notes in mathematics 682, Springer-Verlag 1978.

[3] M.S. Kirdar, T.H.R. Skyrme, On an identity relating to partitions and repetitions of parts. Canad. J. Math. 34 (1982), 194-195.

[4] B. Külshammer, J.B. Olsson, G.R. Robinson, Generalized blocks for symmetric groups. Invent. Math. 151 (2003), 513-552.

[5] J. Müller, On a remarkable combinatorial property, J. Combin. Theory Ser. A 101 (2003), 271-280.

[6] F.W. Schmidt, R. Simion, On a partition identity. J. Combin. Theory Ser. A 36 (1984), 249-252.

[7] R.P. Stanley, Enumerative combinatorics. Vol. 1. Cambridge Studies in Advanced Mathematics, 49. Cambridge University Press, 1997. 